\title{
Color centres in diamond from single photon sources to ODMR in cells
}

E. Moreva, P. Traina, J. Forneris, S. Ditalia Tchernij, F. Picollo, et al.

E. Moreva, P. Traina, J. Forneris, S. Ditalia Tchernij, F. Picollo, I. P. Degiovanni, V. Carabelli, P. Olivero, M. Genovese, "Color centres in diamond from single photon sources to ODMR in cells," Proc. SPIE 10733, Quantum Photonic Devices 2018, 1073304 (11 September 2018); doi: 10.1117/12.2323102

Event: SPIE Nanoscience + Engineering, 2018, San Diego, California, United States 


\title{
Color centres in diamond from Single Photon Sources to ODMR in cells
}

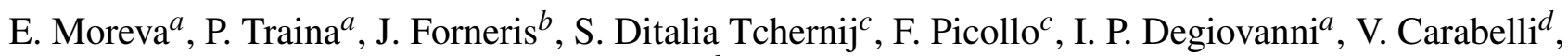 \\ P. Olivero ${ }^{c, b}$ and M. Genovese ${ }^{a}$ \\ ${ }^{a}$ Istituto Nazionale di Ricerca Metrologica (INRIM), Strada delle cacce 91, Torino, Italy; \\ ${ }^{b}$ Istituto Nazionale di Fisica Nucleare (INFN) Sez. Torino,Torino,Italy; \\ ${ }^{c}$ Physics Department and NIS Inter-departmental Centre - University of Torino,Torino,Italy; \\ ${ }^{d}$ Department of Drug Science and Technology - University of Torino,Torino,Italy.
}

\begin{abstract}
Color centres in diamond represent a very interesting system for realizing single photon emitters, even at room temperature, in particular are attracting an ever-growing interest in quantum optics, quantum information and quantum sensing, due to their appealing photo-physical properties combined with ease of access and manipulation in a solid state system characterized by high transparency and structural stability. Literally hundreds of optically active color centers can be created and controlled in the diamond matrix, to be employed either as bright and stable single-photon sources or individual spin systems with optical readout, with record performances even at room temperature. In concurrence with the remarkable results obtained at the state of the art on the exploitation of the unique properties of the negatively-charged nitrogen-vacancy complex (NV), new and appealing color centers are continuously being discovered and characterized. In the present contribution, the most recent results obtained by a collaboration among the Italian National Institutes of Metrologic Research (INRiM), the University of Torino and the Italian National Institutes of Nuclear Physics (INFN) will be overviewed and critically assessed in their future perspectives.
\end{abstract}

Keywords: color centers in diamond, single photon sources, optically detected magnetometry

\section{INTRODUCTION}

Among the several instances in wihich quanutm light has demonstrated to be a fruitful resource for the implementation of enhanced measurements ${ }^{1,2}$ (as in interferometry, ${ }^{3,4}$ biological particle tracking, ${ }^{5}$ phase contrast microscopy, ${ }^{6}$ quantum imaging, ${ }^{7,8}$ quantum illumination ${ }^{9}$ and super-resolved wide-field ${ }^{10,11}$ and confocal ${ }^{12}$ microscopy), here we review some very recent applications of color centers in diamond for the implementation of experiments ranging from the realization of single-photon sources (SPS) to biosensing applications exploiting the process of Optically Detected Magnetic Resonance (ODMR), realized in a collaboration among the Italian National Institutes of Metrologic Research (INRiM), the University of Torino and the Italian National Institutes of Nuclear Physics (INFN). The work is structured as follows: in Sec. 1 the efforts for the realization of novel SPSs based on Sn- and He-related color centers ${ }^{13,14}$ in diamond will be described; In Sec. 2, the results on the implementation of an electrically-pumped ${ }^{15}$ SPS based on NV centers will be shown; Sec. 3 reports on a study ${ }^{16}$ for the characterization of the non-classicality in the emission of optical sources by means of a recently proposed criterion. ${ }^{17}$ Finally, in Sec. 4, preliminary studies ${ }^{18}$ towards the application of ODMR measurements in living cells exploiting NV centers in fluorescent nanodiamonds are described.

Further author information: (Send correspondence to M. Genovese)

M. Genovese: E-mail: m.genovese@inrim.it, Telephone: +39 (0)11 3919253

E. Moreva: e.moreva@inrim.it

P. Traina: p.traina@inrim.it

J. Forneris: forneris@to.infn.it

S. Ditalia Tchernij: sviatoslav.ditaliatchernij@unito.it

F. Picollo: federico.picollo@unito.it

I. P. Degiovanni: i.degiovanni@inrim.it

V. Carabelli: valentina.carabelli@unito.it

P. Olivero: paolo.olivero@unito.it

Quantum Photonic Devices 2018, edited by Cesare Soci, Mario Agio, Kartik Srinivasan, Proc. of SPIE Vol. 10733, $1073304 \cdot$ (c) 2018 SPIE · CCC code: 0277-786X/18/\$18 · doi: 10.1117/12.2323102 


\begin{tabular}{|l|c|c|}
\hline Center & main emission $(\mathrm{nm})$ & lifetime (ns) \\
\hline $\mathrm{Sn}$ & 620.3 & 6 \\
\hline $\mathrm{He} 1$ & 535.2 & 29 \\
\hline $\mathrm{He} 2$ & 559.7 & 100 \\
\hline
\end{tabular}

Table 1. Comparison of the main photo-physical properties of Sn- and He-related centers.

\section{CREATION AND PHOTO-PHYSICAL CHARACTERIZATION OF NOVEL COLOR CENTER BASED ON HE- AND SN-RELATED IMPURITIES IN THE DIAMOND LATTICE}

Here we report on the joint efforts by INRIM, INFN and University of Torino for the creation and characterization of novel single photon-emitters based on not-yet-explored impurities in diamond lattice.

As a first instance, optical centers associated to tin ( $\mathrm{Sn}$ ) were observed in single crystal diamond fabricated upon ion implantation and susequent annealing. Photo-luminescence (PL) characterization at the ensemble level revealed peaks in the emission at $593.5 \mathrm{~nm}, 620.3 \mathrm{~nm}, 630.7 \mathrm{~nm}, 646.7 \mathrm{~nm}$ after excitation at $532 \mathrm{~nm}$. A the single-photon level, PL measurements were performed in INRIM single-photon-sensitive confocal microscope, ${ }^{19}$ whose output was connected to a Hanbury-Brown and Twiss (HBT) interferometer. ${ }^{20}$ Photon were collected in a 580-638 nm spectral window and several isolated emitters, with similar spectral features and non-classical behavior, were observed. The attribution of the PL signal to tin is derived by the main emission line at $620.3 \mathrm{~nm}$, while the non-classical emission properties were studied by means of second-order autocorrelation function $\left(g^{(2)}(t)\right)$ sampled via HBT interferometry. The low value of $g^{(2)}(0)=0.29 \pm 0.02$, even without backgound light subtraction, is definitely compatible with the radiation by a single emitter. Three-level emission structure was assessed with radiative lifetime of $6 \mathrm{~ns}$ and the presence of a shelving state (accompanied by emission blinking at high excitation powers). Polarized light absorption (extinction ratio: 45\%) and saturation in the PL emission (above $1 \mathrm{Mcps}$ ) were also observed. A more detailed description of this experiment can be found elsewhere. ${ }^{13}$

Another interesting, recently observed, family of optically active centers is related to Helium (He). In this case, an extensive study of samples incorporating Helium realated centers was performed and their photo-physical properties were investigated as a function of several parameters (temperature, excitation wavelength amd intensity of external electrical fields). ${ }^{14}$ In particular, emission lines at $535.2 \mathrm{~nm}(\mathrm{He} 1)$ and $559.7 \mathrm{~nm}(\mathrm{He} 2)$ were observed in three samples after $1.3 \mathrm{MeV}$ $\mathrm{He}^{+}$implantation. The mean emission lifetimes for the two PL lines were measured as, respectively, $\tau_{H e 1}=(29 \pm 5) \mathrm{ns}$ and $\tau_{H e 2}=(106 \pm 10) \mathrm{ns}$.

The main photo-physical properties of the described centers are summarized in Table I.

\section{ELECTRICAL CONTROL AND STIMULATION OF NITROGEN-VACANCY CENTERS IN BULK SINGLE-CRYSTAL DIAMOND BY MEANS OF INTEGRATED GRAPHITIC ELECTRODES}

In this section, we report on the realization of a non-classical light emitting diamond-based device with sub-superficial graphitic electrodes for the electrical excitation of single-photon emitters based on $\mathrm{NV}^{0}$ color centers. This experiment, ${ }^{15}$ which is the first instance of investigation of non-classical light electroluminescence from an electrical structure other than a p-i-n junction, provides an insight into the emission mechanism independently of the specific characteristics of the device under test. This experiment was performed on a type-IIa single-crystal Element Six CVD diamond sample ("detector grade": $[N]<5 \mathrm{ppb}$ and $[B]<1 \mathrm{ppb}$, respectively) in which bulk two parallel sub-superficial graphitic microelectrodes were directly written by raster-scanning a $10 \mu \mathrm{m}$ focused $6 \mathrm{MeV} \mathrm{C}^{3+}$ beam. The light emission characterization was performed by means of photoluminescence (PL) and EL mapping, using a dedicated single-photon-sensitive confocal microscope. In PL measurements, the device was not electrically pumped and the color centers were excited with continuous laser light $(\lambda=532 \mathrm{~nm}, P=0.4 \mathrm{~mW})$. In EL measurements, the laser pump was stopped and elecrtical current was injected in the inter-electrode gap. PL and EL maps were acquired at $\approx 3 \mu \mathrm{m}$ below the diamond surface. Both PL and EL $(240 \mathrm{~V}$ bias voltage) spectral characterization of the center emission was performed in the $550-800 \mathrm{~nm}$ range, highlighting the emission from $\mathrm{NV}^{0}$ centers, with a zero phonon line (ZPL) at $\lambda=575 \mathrm{~nm}$ together with phonon sidebands at higher wavelengths. Second-order auto-correlation measurements were performed adopting a HBT interferometry, demontrating the single-photon emission character of the fabricated device $\left(g^{(2)}(0) \approx 0.46\right.$, after dark counts subtraction). This technique in perspective could allow to realize arrays of electrically-stimulated single-photon sources of potential interest in quantum technology. 


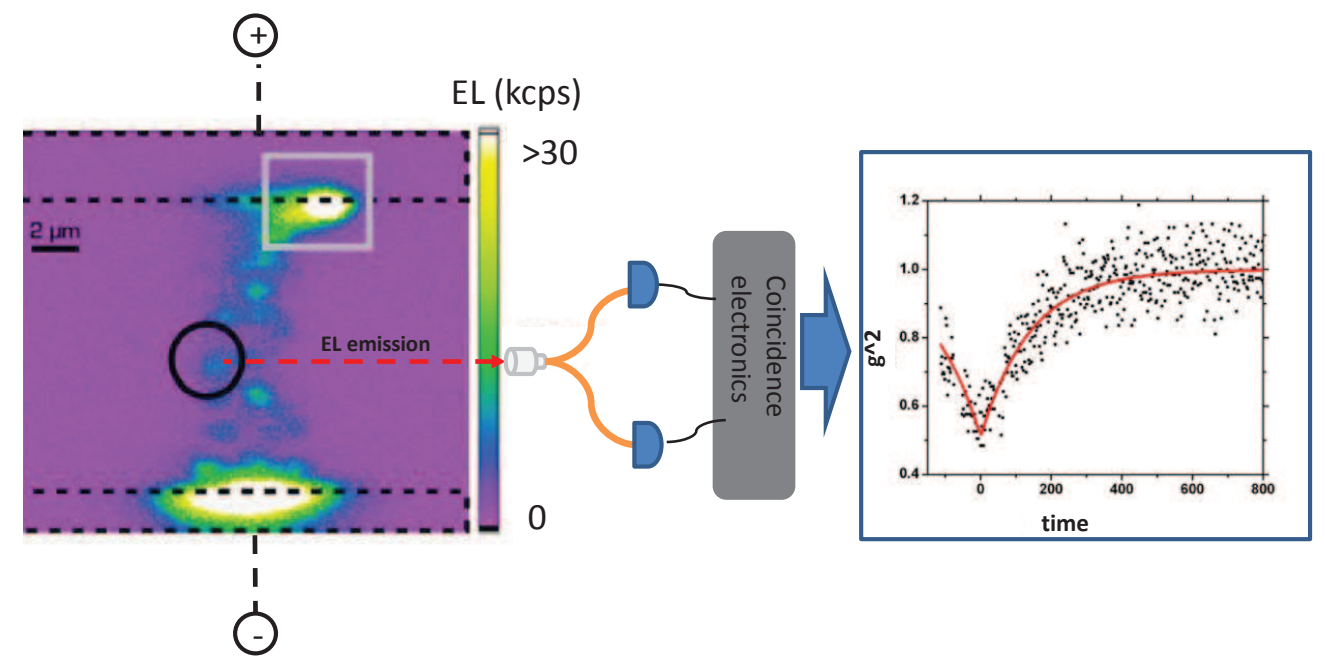

Figure 1. Realization of a non-classical light emitting diamond-based device with sub-superficial graphitic electrodes for the electrical excitation of single-photon emitters based on $\mathrm{NV}^{0}$ color centers. More details can be found in the original publication. ${ }^{15}$

\section{NOVEL CRITERIA TO ASSESS NON-CLASSICAL BEHAVIORS IN THE FLUORESCENCE EMISSION FROM ENSEMBLES OF COLOR CENTERS IN DIAMOND}

In the following, we briefly report on the experimental application ${ }^{16}$ of a criterion ${ }^{17}$ to directly detect non-classical emission from ensembles of SPSs based on Nitrogen-Vacancy (NV) centers in nano-diamond observed by means of a confocal microscope coupled to four non-PNR single-photon detectors in a detector-tree ${ }^{21}$ configuration (Fig. 4).

The proposed criterion is compared to the generalized $g^{(N)}(0)$ function that can be expressed in terms of detection probabilities as:

$$
g^{(N)}(0)=\frac{P_{\otimes N}}{\prod_{i=1}^{N} P_{[i]}},
$$

where $P_{\otimes N}$ is the probability of $N$-fold coincidence at the output of the detector-tree, and $P_{[i]}$ is the click probability for the $i$-th detector. Usually, the condition $g^{(N)}(0)=1$ is adopted to distinguish between classical and non-classical states, while ideally $g^{(N)}(0)=0$ for any order of $N$ for single-photon states. The nonclassicality criterion considered in this section ${ }^{17}$ is expressed by the fact that, for any classical system,

$$
\Theta^{(N)}(0)=\frac{P_{\otimes N}^{\prime}}{\prod_{i=1}^{N} P_{[i]}^{\prime}}>1,
$$

where, $P_{[i]}^{\prime}$ is the no-click probability at the $i$-th detector and $P_{\otimes N}^{\prime}$ is the probability that all the $N$ detectors of the detectortree do not click.

The comparison of $g^{(2)}$ and $\Theta^{(2)}$ on the signals radiated by several ensembles of single-photon emitters with different levels of background Poissonian noise in synthetic Ib nanodiamond (ND) powders was performed. 


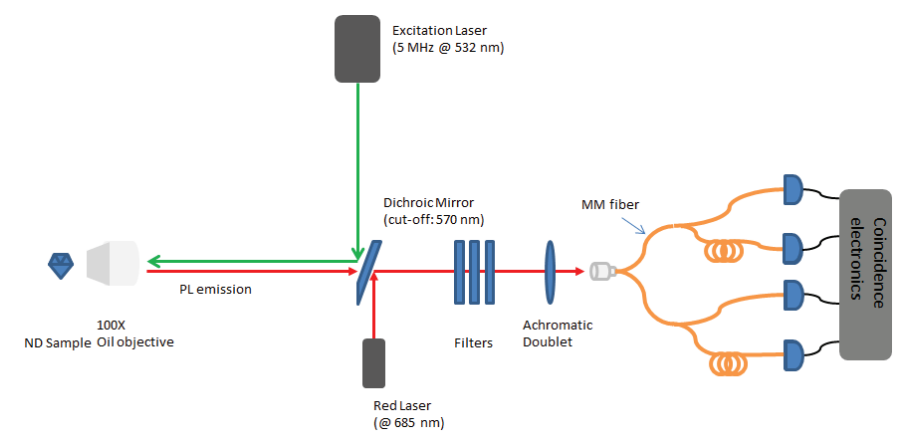

Figure 2. Experimental setup for the characterization of nonclassicality of of SPSs via a novel criterion. ${ }^{17}$ For further detatils on the single-photon sensitive confocal microscope coupled to a detector-tree or the used sample, please refer to the original publication ${ }^{16}$

\begin{tabular}{|l|l|l|l|}
\hline$g^{(2)}(0)$ viol. & Noise Level 1 & Noise Level 2 & Noise Level 3 \\
\hline \hline I-1 & $0.61 \pm 0.03$ & $0.32 \pm 0.04$ & $0.12 \pm 0.02$ \\
\hline I-2 & $0.168 \pm 0.004$ & $0.139 \pm 0.006$ & $0.088 \pm 0.011$ \\
\hline I-3 & $0.34 \pm 0.01$ & $0.254 \pm 0.017$ & $0.135 \pm 0.016$ \\
\hline
\end{tabular}

Table 2. Table of the experimental violations of classicality of $g^{(2)}(0)$.

In particular, the measurement of $g^{(2)}$ and $\Theta^{(2)}$ for the characterization of three fluorescent clusters of NV centers (dubbed I-1,I-2,I-3) in a nano-diamonds sample was performed by sampling the coincidences at the output of the detectortree in a $40 \mathrm{~ns}$ coincidence window (compatible with the lifetime of the centers, around $25 \mathrm{~ns}$ ). A laser source at 685 $\mathrm{nm}$, directly coupled in the pinhole of the microscope, was used to simulate Poissonian noise. The robustness of the two parameters against noise was tested by performing measurements on each cluster in the absence of noise and in the presence of two different values of intensity of the noise source. These two noise levels, estimated in terms of counts/s observed by the HBT system as a whole, in the absence of single-photon source emissions were 10000 counts/s and 25000 counts/s.

Each measurement was the average of 10 runs, coincidences registered in every two-fold combination of the detectortree branches, of $200 \mathrm{~s}$. Excitation rate was $5 \mathrm{MHz}$.

Tab.s $2 \& 3$ report the experimental violations of classicality $\left(1-g^{(2)}(0), 1-\Theta^{(2)}(0)\right)$.

As theoretically expected, ${ }^{17}$ the experimental data show that: 1) the $\Theta$ parameter estimation is more robust against Poissonian noise since the three $\Theta^{(2)}$ values for each cluster are totally compatible, while $g^{(2)}$ differs considerably for varying noise levels; 2) the classicality violation is even higher when the source is not a single emitter but instead an ensemble of them. in fact: the classicality violationin terms of $\Theta^{(2)}$ is larger for clusters characterized by a greater $g^{(2)}$ value with respect to the others (measured in the same conditions). For this reasons this $\Theta$ allows detecting non-classical behavior of quantum emitters without the necessity of isolating a single one. On the other hand, the $\Theta^{(2)}$ depends strongly on the efficiency of the channel, and this is a disadvantage with respect to $g^{(2)}$.

\begin{tabular}{|l|l|l|l|}
\hline$\Theta^{(2)}(0)$ viol. & Noise Level 1 & Noise Level 2 & Noise Level 3 \\
\hline \hline I-1 & $(4.3 \pm 1.2) \mathrm{E}-6$ & $(4.3 \pm 1.2) \mathrm{E}-6$ & $(4.1 \pm 1.2) \mathrm{E}-6$ \\
\hline $\mathrm{I}-2$ & $(11 \pm 3) \mathrm{E}-6$ & $(11 \pm 3) \mathrm{E}-6$ & $(9 \pm 2) \mathrm{E}-6$ \\
\hline $\mathrm{I}-3$ & $(8 \pm 2) \mathrm{E}-6$ & $(8 \pm 2) \mathrm{E}-6$ & $(8 \pm 2) \mathrm{E}-6$ \\
\hline
\end{tabular}

Table 3. Table of the experimental violations of classicality of $\Theta^{(2)}(0)$. 
In this experiment the analysis was limited to the second order of correlation of $\theta$ and $g$ functions, but it can be generalized to any order. Recently another application of this criterion has been independently demonstrated exploiting trapped ions. ${ }^{22}$

\section{TOWARDS ODMR MEASUREMENTS IN LIVING CELLS}

Nitrogen-vacancy (NV) centers in diamond provide a stable photoluminescence (PL), resistance to bleaching or quenching phenomena, low cytotoxicity, have wide optical transparency window and extreme mechanical properties. In addition, they have a spin triplet ground state, which is optically addressable and coherently controllable by microwaves fields. This feature opens new perspective in detection of weak electromagnetic fields and small temperature variations within the biological samples with unprecedented spatial resolution and sensitivity, by means of Optically Detected Magnetic Resonance (ODMR). ${ }^{23-26}$ ODMR technique is based on the dependence of intensity of PL on the two electronic spin states of NV center in diamond. After initializing the NV center in the bright state applying a 532nm laser, by stimulating the center (always while optically exciting it) with a microwave signal at variable frequency one observes a dip in the PL signal corresponding to the transition frequency $(\sim 2.87 \mathrm{GHz})$ between the two spin states. If a magnetic field is applied to the sample the spin state splits into two by Zeeman effect, thus one observes two dips in the PL vs frequency diagram, with a distance between them equal to $2 g_{e} \mu_{b} B_{z} / h$. Any additional external magnetic/electrical field changes the distance between the dips and can be detected with a very high sensibility, so very faint magnetic fields (and thus weak electrical currents) can be measured in living cells in a non-disruptive way. Unfortunately, the magnetic sensibility is limited by different factors, like: temperature and magnetic field fluctuation, NV spin coherence time, intensity of laser and microwave field, and fundamental quantum shot-noise. In order to protect spin coherence of NVs different protocols based on pulsed dynamical decoupling were suggested. The thermal drift can be controlled by monitoring the transition frequency, which depends on temperature. In a recent work ${ }^{18}$ we applied the ODMR technique to detect signals from nanodiamonds (ND) internalized into hippocampal neurons (Fig. 3). ND internalization Was assessed by means of commercial confocal microscope. Embryo hippocampal neurons were incubated with $40 \mu \mathrm{g} / \mathrm{ml}$ ND. Successively, the cytoplasmic membrane labelling dye (CellBrite green cytoplasmic membrane dye, Biotium) was added to the medium ( $24 \mathrm{~h}$ later). This allowed to identify the intracellular membranes (stained in green) together with the internalized NDs, characterized by a red fluorescent emission. Representative images are shown in Fig.3. The ODMR technic was demonstrated on the dedicated setup for biosensing measurements Fig. 4, i. e. a single-photon-sensitive confocal microscope integrated to commercial inverted microscope Olimpus IX73, the excitation light being provided by a solid state laser at $532 \mathrm{~nm}$. A dichroic beamsplitter (long-pass at $570 \mathrm{~nm}$ ) reflected the excitation light $(3 \mathrm{~mW}$ maximum) inside the air objective (Olympus, $60 \times, \mathrm{NA}=0.9)$ focusing inside the sample and transmitted the PL towards the detecting apparatus. A microwave field with power Pmw $=-25 \mathrm{dBm}$ was transmitted via micro strip antenna, the experimental control and data acquisition were performed using the labview software suite. During an ODMR experiment, the sample is continuously illuminated and irradiated by microwaves at a frequency not far from the spin resonance. The frequency is slowly scanned while the photoluminescence (PL) is being monitored. When approaches the resonance frequency, the photoluminescence will be diminished and a dip corresponding to the transition frequency $(2.87 \mathrm{GHz})$ between the two spin states can be observed Fig. 4 (inset). Finally, combining patch-clamp electrophysiology together with microelectrode arrays recordings, was confirmed that these cells were not affected by the implementation of the measurement protocol, which apart from the NDs internalization is based on the application of both MW fields and laser illumination. Since each NV center can be regarded as an atomic-sized magnetic field sensor that can be read-out optically using ODMR, in perspective their excellent magnetic field sensitivity can be applied to perform sensing protocols in order to detect the action potential propagation on the plasma membrane.

\section{CONCLUDING REMARKS}

In this work we described the most recent results obtained in a collaboration among the Italian National Institutes of Metrologic Research (INRiM), the University of Torino and the Italian National Institutes of Nuclear Physics (INFN). Our results prove the advantage of exploiting quantum features of NV centers in diamond for advancement in several fields ranging from quantum optics to biosensing, encouraging further investigation.

\section{ACKNOWLEDGMENTS}

This research activity was supported by the following projects: EMPIR Project. No. 14IND05-MIQC2, EMPIR project 17FUN01 "BeCOMe", "DIESIS" project funded by the Italian National Institute of Nuclear Physics (INFN) - CSN5 within 
the Young research grant scheme.

\section{REFERENCES}

1. V. Giovannetti, S. Lloyd, and L. Maccone, Science 306, 1330 (2004).

2. K. Banaszek et al., Nature Phot. 3, 673 (2009).

3. J. Abadie et al., Nature Phys 7, 962 (2011).

4. I. Ruo-Berchera, I. P. Degiovanni, S. Olivares, and M. Genovese, Phys. Rev. Lett. 110, 213601 (2013).

5. M. A. Taylor et al., Nature Photonics 7, 229(2013).

6. Takafumi Ono, Ryo Okamoto and Shigeki Takeuchi, Nat. Comm. 4, 2426 (2013).

7. G. Brida, M. Genovese, and I. Ruo-Berchera, Nat. Photonics 4, 227 (2010).

8. G. Brida et al., Phys. Rev. A 83, 063807 (2011).

9. M.Genovese, Journal of Optics 18, 073002 (2016).

10. O. Schwartz and D. Oron, Phys. Rev. A 85, 033812 (2012).

11. O. Schwartz et al., Nano Lett. 13, 5832 (2013).

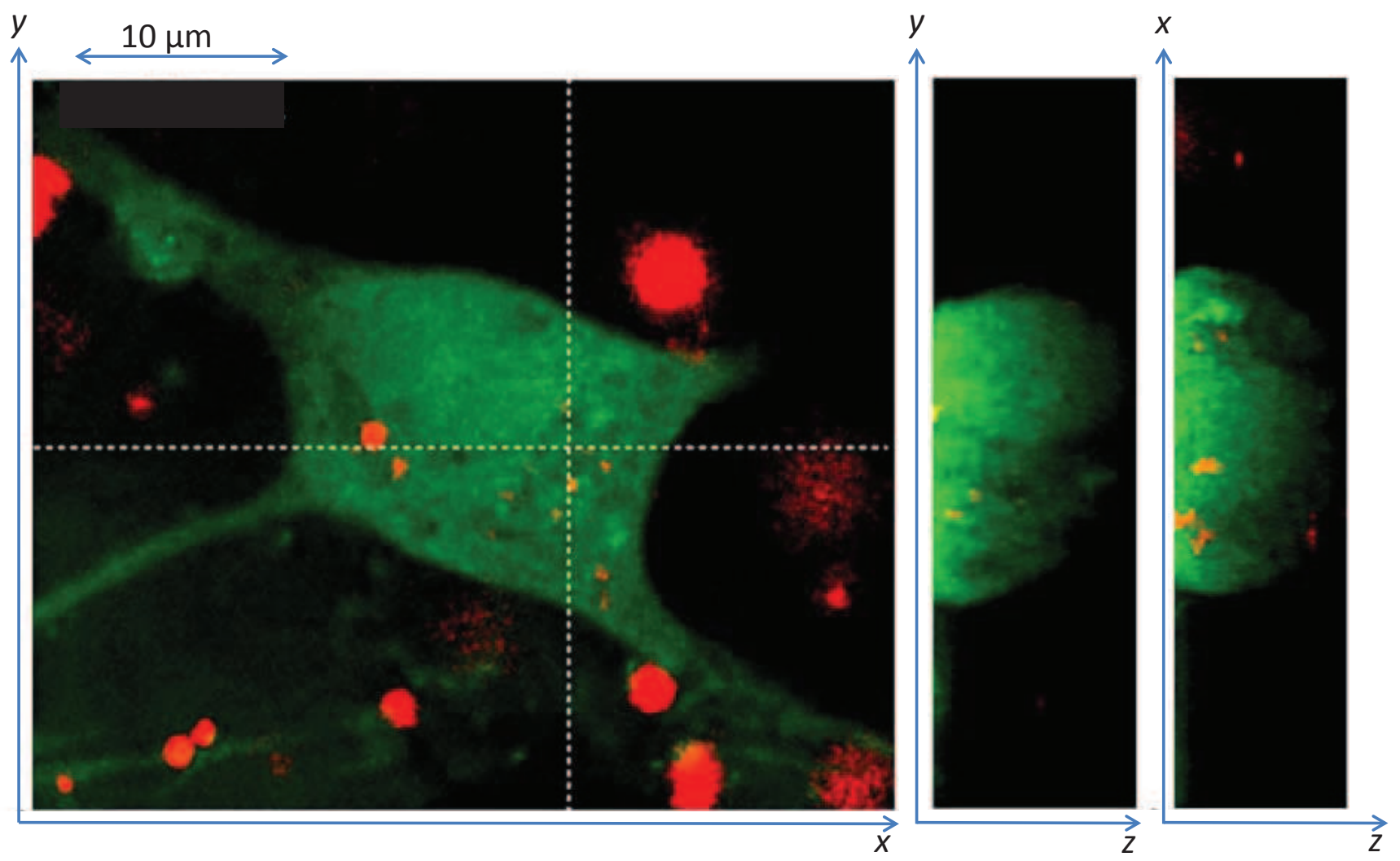

Figure 3. Confocal fluorescence micrograph of cultured hippocampal neurons, exposed to $40 \mu \mathrm{g} / \mathrm{ml}$ ND ND for 2 days, and stained in green with the cytoplasmic labelling dye. Red emission is from NDs. The entire field and cross-sections $(X Z$ and $Y Z)$ were shown. For futher details, please refer to the original publication ${ }^{18}$ 
12. D. Gatto Monticone, et al., Phys. Rev. Lett. 113, 143602 (2014).

13. S. Ditalia Tchernij et al., ACS photonics 4, 2580-2586 (2017).

14. G. Prestopino et al., Appl. Phys. Lett. 11, 111105 (2017).

15. J. Forneris, P. Traina, D. Gatto Monticone, G. Amato, L. Boarino, G. Brida, I. P. Degiovanni, E. Enrico, E. Moreva, V. Grilj, N. Skukan, M. Jakšić, M. Genovese, and P. Olivero, Sci. Rep. 5, 15901 (2015).

16. E. Moreva, P. Traina, J. Forneris, et al., Phys. Rev. B 96, 195209 (2017)

17. L. Lachman, L. Slodička, \& R. Filip, Sci. Rep. 6, 19760 (2016).

18. L. Guarina et al, Sci. Rep. 8(1), 2221 (2018).

19. D. Gatto Monticone et al., New J. Phys. 16, 053005 (2014).

20. R. Hanbury-Brown and R. Q. Twiss, Nature 177, 27 (1956).

21. H. Paul, P. Torma, T. Kiss, I. Jex, Phys. Rev. Lett. 76, 2464 (1996); L. A. Jiang, E. A. Dauler, and J. T. Chang, Phys. Rev. A 75, 062325 (2007); V. Schettini et al., IEEE Quant. Electron. Sel. Topics. 13, 978 (2007); A. Divochiy et al., Nature Photon. 2, 302 (2008); E. A. Dauler et al., J. Mod. Opt. 56, 364 (2009); D. Rosenberg et al., Opt. Exp. 21, 1440 (2013); K. Banaszek and I. A. Walmsley, Opt. Lett. 28, 52 (2003); M. J. Fitch, B. C. Jacobs, T. B. Pittman, and J. D. Franson, Phys. Rev. A 68, 043814 (2003); D. Achilles, C. Silberhorn, C. Sliwa, K. Banaszek, and I. A. Walmsley, Opt. Lett. 28, 2387(2003).

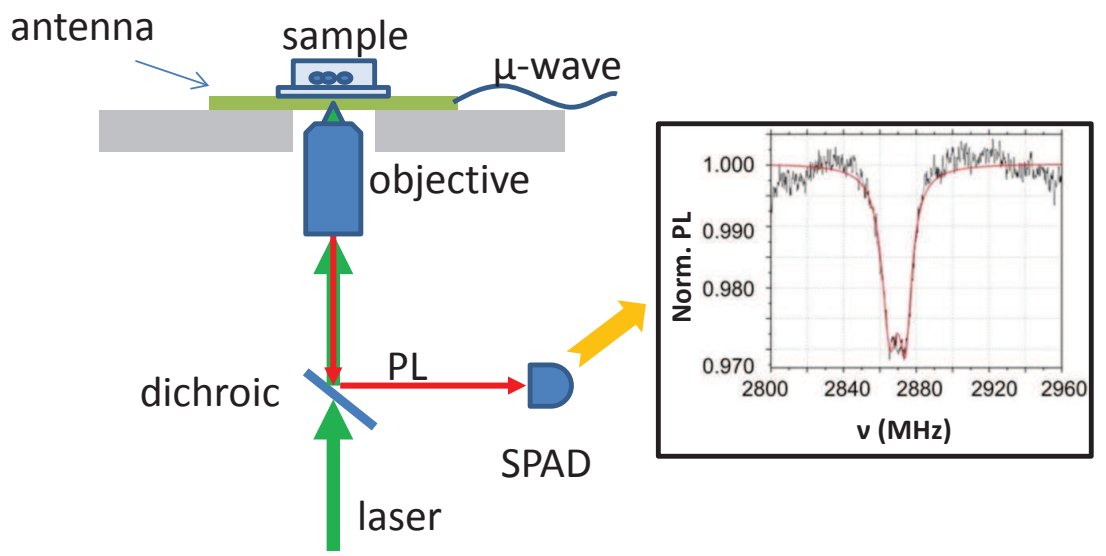

Figure 4. Schematics of the confocal setup for ODMR measurements. Inset: ODMR signal detected from NV centers in nanodiamonds internalized in a neural cell.For futher details, please refer to the original publication ${ }^{18}$ 
22. P. Obšil et al., Phys. Rev. Lett. 120, 253602 (2018).

23. Barry, J. F. et al. Optical magnetic detection of single-neuron action potentials using quantum defects in diamond. Proceedings of the National Academy of Sciences 113, 1413314138, (2016);

24. Glenn, D. R. et al. Single-cell magnetic imaging using a quantum diamond microscope. Nat Meth 12, 736738, (2015).

25. Kucsko, G. et al. Nanometre-scale thermometry in a living cell. Nature 500, 5458, (2013).

26. Hemelaar, S. R. et al. Nanodiamonds as multi-purpose labels for microscopy. Scientific reports 7, 720, (2017). 